\title{
Effects of Transplanted Human Cord Blood-Mononuclear Cells on Pulmonary Hypertension in Immunodeficient Mice and Their Distribution
}

\author{
Mikio Sugano ${ }^{1}$; Homare Yoshida ${ }^{1}$; Hirotsugu Kurobe ${ }^{1}$; Hiroki Arase ${ }^{1}$; Hajime Kinoshita ${ }^{1}$; Takashi Kitaichi $^{1}$; \\ Noriko Sugasawa ${ }^{1}$, Soichiro Nakayama ${ }^{2}$; Kazuhisa Maeda ${ }^{2}$; Minoru Irahara² ${ }^{\text {; }}$ and Tetsuya Kitagawa ${ }^{1}$ \\ Department of Cardiovascular Surgery ${ }^{1}$ and Gynecology ${ }^{2}$, Graduate School of Biomedical Sciences, Tokushima University, Tokushima, Japan
}

\begin{abstract}
Objectives : To investigate the effects of human umbilical cord blood -derived mononuclear cell (hUCB MNC) transplantation on pulmonary hypertension (PH) induced by monocrotaline (MCT) in immunodeficient mice and their distribution. Methods : MCT was administered to BALB/c Slc-nu/nu mice, and PH was induced in mice 4 weeks later. Fresh hUCB-MNCs harvested from a human donor after her delivery were injected intravenously into those PH mice. The medial thickness of pulmonary arterioles, ratio of right ventricular to septum plus left ventricular weight $(\mathrm{RV} / \mathrm{S}+\mathrm{LV})$, and ratio of acceleration time to ejection time of pulmonary blood flow waveform (AT/ET) were determined 4 weeks after hUCB-MNC transplantation. To reveal the incorporation into the lung, CMTMR-labeled hUCB-MNCs were observed in the lung by fluorescent microscopy. DiR-labeled hUCBMNCs were detected in the lung and other organs by bioluminescence images. Results : Medial thickness, RV/S+ LV and AT/ET were significantly improved 4 weeks after hUCB-MNC transplantation compared with those in mice without hUCB-MNC transplantation. CMTMR-positive hUCB-MNCs were observed in the lung 3 hours after transplantation. Bioluminescence signals were detected more strongly in the lung than in other organs for 24 hours after transplantation. Conclusions : The results indicate that hUCB-MNCs are incorporated into the lung early after hUCB-MNC transplantation and improve MCT-induced PH. J. Med. Invest. 64 : 43-49, February, 2017
\end{abstract}

Keywords : pulmonary hypertension, cellular therapy, umbilical cord blood

\section{INTRODUCTION}

There are many patients who are suffer from pulmonary artery hypertension (PAH) due to pulmonary vascular obstructive disease or limited pulmonary vasculature with or without congenital heart defects. Although there has been significant progress in the development of medication for PAH over the past two decades, most patients with severe PAH have shown little favorable response to any medication and their prognosis has been very poor.

Results of recent studies using animal models with hypoxia- or monocrotaline (MCT) -induced pulmonary hypertension ( $\mathrm{PH})$ and results of a human pilot study have shown that cellular therapy using endothelial progenitor cells harvested from large quantities of peripheral blood or bone marrow (BM) blood contributed to pulmonary vascular remodeling, thus providing some useful clues for PAH treatment (1-4). Also, our previous study showed that syngeneic BM mononuclear cells improved MCT-induced $\mathrm{PH}$ in mice model (5). However, harvesting these cells from large quantities of peripheral blood or BM blood is very invasive and dangerous for patients with severe $\mathrm{PAH}$, especially for children.

In a recent study, it was shown that a single low dose ( $10^{6}$ cells $)$ of human umbilical cord blood-derived mononuclear cells (hUCBMNCs) administered intravenously to G93A mice with amyotrophic lateral sclerosis delayed symptom progression and modestly

Received for publication March 25, 2016 ; accepted September 19, 2016.

Address correspondence and reprint requests to Tetsuya Kitagawa, MD, $\mathrm{PhD}$, Chairman and Professor, Tokushima University, Graduate School of Biomedical Sciences, Department of Cardiovascular Surgery, 3-18-15 Kuramoto, Tokushima 770-8503, Japan and Fax : +81-88-633-7408. prolonged lifespan (6). It has been shown that human umbilical cord blood contains abundant with hematopoietic stem cells, mesenchymal stem cells, and endothelial progenitor cells that are able to differentiate into nerve cells and endothelial cells $(7-10)$.

Therefore, we hypothesized that hUCB-MNC transplantation may provide a new therapeutic potential for patients with severe $\mathrm{PAH}$ or limited pulmonary vasculature. The aims of this study were to investigate whether hUCB-MNCs themselves rather than hUCBplasma improve $\mathrm{PH}$ in MCT-treated nude mice and to reveal the locations of hUCB-MNCs in the lung.

\section{METHODS}

\section{Animal Preparation}

BALB/c Slc- nu/nu female immunodeficient mice (Japan SLC, Tokyo, Japan) were used at 8 weeks of age. We anesthetized the mice with ketamine $(100 \mathrm{mg} / \mathrm{kg})$ and xylazine $(10 \mathrm{mg} / \mathrm{kg})$. Animal care and procedures were in accordance with the institutional guidelines. This study was approved by a university ethics review board (\#912), and the animal procedures were conformed to the NIH guidelines (Guide for the care and use of laboratory animals).

\section{Pulmonary Hypertension Models}

Mice received monocrotaline (MCT) $\left(\mathrm{C}_{16} \mathrm{H}_{26} \mathrm{NO}_{6}\right.$; Sigma-Aldrich, Lyon, France) treatment. MCT treatment was previously demonstrated to be capable of inducing $\mathrm{PH}$ in mice $(5,11)$.

MCT was suspended in $0.1 \mathrm{~N} \mathrm{HCl}$, which was adjusted to $\mathrm{pH} 7.0$ with $\mathrm{NaOH}$ and diluted in phosphate-buffered saline (PBS). A single injection of MCT $(80 \mathrm{mg} / \mathrm{kg}$ body weight) into the peritoneum 
was performed. That dosage did not cause toxic effects to the liver. The completion rate of $\mathrm{PH}$ lesion of $90 \%$ to $100 \%$ and survival rate of PH model mice was $100 \% 4$ weeks after MCT injection.

\section{Umbilical Cord Blood Mononuclear Cell Isolation}

Fresh human umbilical cord blood was obtained from human donors with agreement for the harvest shortly after their delivery of healthy children after more than 38 weeks of gestation. The part of this study was performed conform the declaration of Helsinki and was approved by a university ethics review board (\#912). HUCBMNCs were isolated and enriched using density centrifugation (Ficoll-Paque Plus ; Invitrogen Australia Pty Ltd, Mulgrave, Australia). The number of injected hUCB-MNCs was based on results of previous studies using unfractionated $\mathrm{BM}$ cells in a mouse or rat PH model $\left(5 \times 10^{6}\right.$ to $1 \times 10^{8}$ cells $)(5,12)$. HUCB contained $1.02 \%$ of CD34+, CD45 + and CD133 + progenitor cells, which were suspended in PBS and adjusted to $1 \times 10^{7}-\mathrm{MNCs} / 0.25 \mathrm{ml}$ with a concentration of $2.57 \%$ of CD34+, CD45+ and CD133 + progenitor cells. The hUCB-MNC suspension fluid was delivered into MCT-treated $\mathrm{PH}$ mice through the orbital vein by using $27 \mathrm{G}$ needle.

\section{Study Protocol}

Vehicle, hUCB-MNCs or hUCB- plasma were injected to $\mathrm{PH}$ mice after MCT-treatment. The mice were divided into four groups : 1) vehicle-injected mice (control; $n=5) ; 2$ ) MCT-treated mice $(\mathrm{PH}$ model $; \mathrm{n}=5)$; 3 ) MCT-treated mice 4 weeks after hUCBMNC transplantation (CB-cell ; n=5) ; and 4) MCT-treated mice 4 weeks after hUCB-plasma injection (CB-plasma; $n=5$ ) (Fig. 1). All mice were sacrificed 4 weeks after vehicle, hUCB-MNCs or hUCB-plasma injection, and heart and lung specimens were harvested.

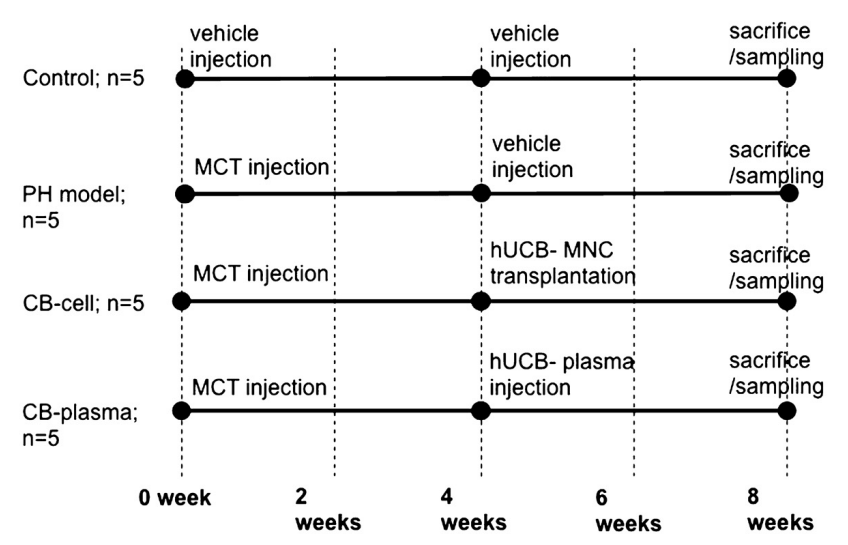

Fig. 1 Study protocol.

$\mathrm{PH}$, pulmonary hypertension ; $\mathrm{CB}$, cord blood ; $\mathrm{MCT}$, monocrotaline ; hUCB-MNCs, human umbilical cord blood-derived mononuclear cells ; n, number of mice.

\section{Histologic Evaluation}

The lungs were perfused through the heart with PBS solution and fixed with $4 \%$ paraformaldehyde in PBS solution. Four-micrometer-thick lung sections were cut and stained with elastica van Gieson after fixation and paraffin embedding. In each mouse, all intraacinar vessels accompanying the alveolar ducts or alveoli were examined. Medial thickness of the small pulmonary arteriole (50 to $100 \mu \mathrm{m}$ in diameter) was measured under a light microscope $(\times 40$ magnification), and percent medial thickness was calculated as medial thickness divided by diameter of the pulmonary artery $\times 100$.

\section{Assessment of Pulmonary Hypertension}

After the heart had been removed, the right ventricle (RV) was dissected from the left ventricle+septum $(\mathrm{LV}+\mathrm{S})$, and the dissected samples were weighed. The ratio of RV/S+LV weight was determined by a previously described method for estimation of RV hypertension caused by $\mathrm{PH}(5,13)$.

Assessment of Pulmonary Hypertension by Using an Echocardiogram

Transthoracic closed-chest echocardiography was performed in blinded fashion using a mechanical transducer centered on 12 $\mathrm{MHz}$ (PLT-1202S, Toshiba, Toyo, Japan). Two-dimensional images of the pulmonary infundibulum were obtained from the parasternal short axis view at the level of the aortic valve, and pulsed-wave Doppler recording of pulmonary blood flow was obtained. By analyzing the waveform, the following variables were measured : pulmonary acceleration time (AT) and right ventricular ejection time (ET). As an index of PAH, we calculated AT/ET as previously described (14).

Assessment of Incorporation of Transplanted Umbilical Cord Blood-derived Mononuclear cells Into the Lung and Other Organs

Two methods were used to determine the incorporation of the transplanted hUCB-MNCs in the lungs of MCT-treated mice early after delivery.

Firstly, hUCB-MNCs were labeled with the vital fluorescent dye chloromethyl tetramethylrhodamine (CMTMR; Molecular Probes Inc., Eugene, OR) and injected into the orbital veins of MCT-treated mice $\left(1 \times 10^{7}\right.$ cells $)$. The mice were sacrificed separately at 10 minutes, 30 minutes, 1 hour, and 3 hours after labeled hUCB-MNC delivery ( $n=5$ /group). Lungs were fresh frozen in OCT compound (Sakura, Torrance, $\mathrm{CA}$ ), and transverse sections $(20 \mu \mathrm{m})$ were taken. CMTMR-labeled hUCB-MNCs were detected by fluorescent microscopy as described previously (15).

Secondly, to reveal the homing of injected hUCB-MNCs to the lungs and elucidate the distribution to other organs, additional MCT-treated mice were analyzed with IVIS $R$ Spectrum (SPI, Tokyo, Japan). HUCB-MNCs $\left(1 \times 10^{7}\right)$ were labeled with XenoLight DiR (SPI, Tokyo, Japan) and delivered into MCT-treated $\mathrm{PH}$ mice through the orbital vein. MCT-treated mice injected with a vehicle were used as controls. The mice were sacrificed, and bioluminescence signals of organs including the lung, heart, liver, spleen and intestine were recorded at 10 minutes, 30 minutes, 1 hour, 3 hours, 6 hours and 24 hours after labeled hUCB-MNC delivery. Bioluminescence records were layered onto a visual photographic image to create composite bioluminescence-photographic images. The fluorescent signals of each organ were analyzed quantitatively by region of interest (ROI) using IVIS $($ spectrum software $(16,17)$.

\section{Statistical Analysis}

Data are presented as means \pm standard deviation. Statistical comparisons were performed using unpaired two-tailed Student's $t$ tests or analysis of variance with Scheffe's test as appropriate, with a probability value of less than 0.05 taken to indicate significance.

\section{RESULTS}

No mice were dead in the acute phase after hUCB-MNC transplantation and no symptom of thrombotic microangiopathy, which is known as a possible complication of bone marrow-MNC transplantation, was observed. 


\section{Histologic Evaluation}

The medial walls of the distal small pulmonary arteries were thickened significantly in $\mathrm{PH}$ mice, and remarkable fibrosis of the adventitia was observed in the $\mathrm{PH}$ mice compared with findings in control mice (Fig. 2). However, these findings were improved significantly 4 weeks after hUCB-MNC transplantation. On the other hand, no improvement was observed in the mice that received hUCB-plasma injection without hUCB-cell injection. The number of small pulmonary arteries decreased in the $\mathrm{PH}$ model (Fig. 2f), compared with that of the control mice (Fig. 2e), and recovered up to the same level as that in the control mice after hUCB-MNC transplantation (Fig. 2g), but not recovered after hUCB-plasma injection without hUCB-cell injection (Fig. 2h).

\section{Improvement of Pulmonary Hypertension After Human Umbilical} Cord Blood-Mononuclear Cell Transplantation

Medial thickness was significantly increased in MCT-treated mice, being consistent with $\mathrm{PH}$, compared with that in control mice $(15.8 \% \pm 4.3 \%$ versus $8.4 \% \pm 3.5 \%$; $<<0.01$; Fig. 3$)$. However, medial thickness was decreased significantly 4 weeks after hUCBMNC transplantation compared with that in $\mathrm{PH}$ mice $(11.5 \% \pm 4.7 \%$ versus $15.8 \% \pm 4.3 \% ; \mathrm{p}<0.01)$. This improvement was not seen in mice that received hUCB-plasma injection without hUCB-cell injection $(15.1 \% \pm 5.4 \%)$.

The $\mathrm{RV} / \mathrm{S}+\mathrm{LV}$ ratio was also increased in MCT mice compared with that in control mice $(0.35 \pm 0.06$ versus $0.29 \pm 0.08 ; \mathrm{p}=0.23)$. On the other hand, the RV/S+LV ratio was reduced significantly 4 weeks after hUCB-MNC transplantation compared with that in PH control mice $(0.27 \pm 0.04$ versus $0.35 \pm 0.06 ; \mathrm{p}<0.05)$ (Fig. 3$)$. No improvement was observed in mice that received hUCB-plasma injection without hUCB-cell injection $(0.31 \pm 0.02 ; \mathrm{p}=0.29)$.

The AT/ET ratio was decreased significantly in $\mathrm{PH}$ mice 4 weeks after MCT injection $(0.36 \pm 0.06$ versus $0.26 \pm 0.02 ; \mathrm{p}<0.01)$ and remained at a similar level 8 weeks after MCT injection $(0.24 \pm$ 0.02) (Fig. 3, 4). However, the AT/ET ratio was increased significantly 4 weeks after hUCB-MNC injection $(0.26 \pm 0.02$ versus $0.35 \pm 0.01 ; \mathrm{p}<0.01)$. On the other hand, the ratio in mice that a)

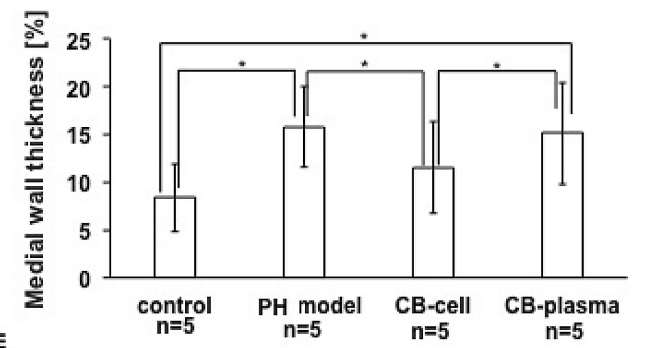

b)

c)
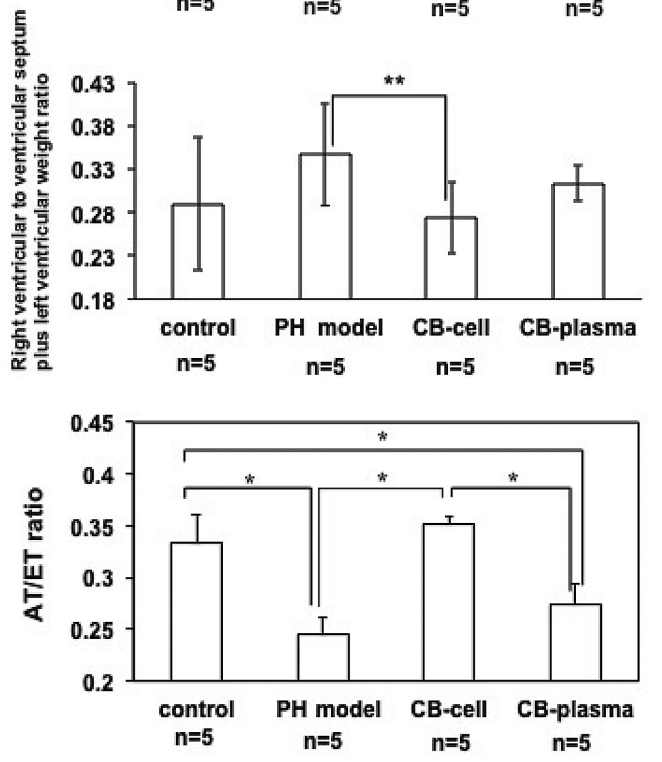

Fig. 3 a) Changes in the percentage of medial wall thickness. b) Changes in $\mathrm{RV} / \mathrm{S}+\mathrm{LV}$ weight ratio. c) Changes in $\mathrm{AT} / \mathrm{ET}$ ratio. $\mathrm{PH}$, pulmonary hypertension ; CB, cord blood ; n, number of mice ; RV/S+LV weight ratio, the right ventricular to ventricular septum plus left ventricular weight ratio ; AT/ET, pulmonary acceleration time to right ventricular ejection time ratio. $(* \mathrm{P}<0.01 ; * * \mathrm{P}<0.05)$ a)

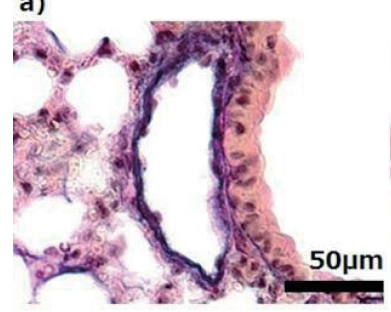

e)

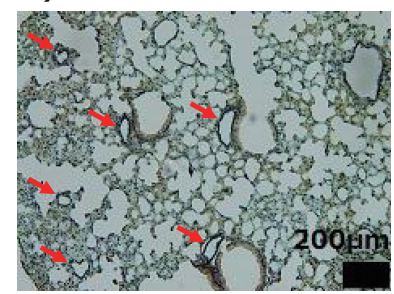

b)

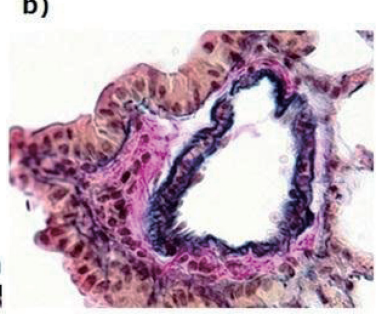

f)

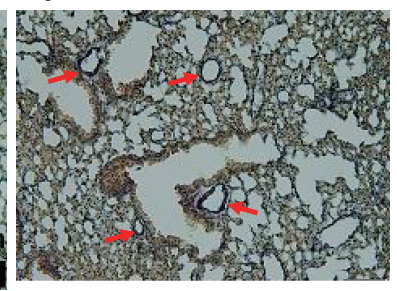

c)

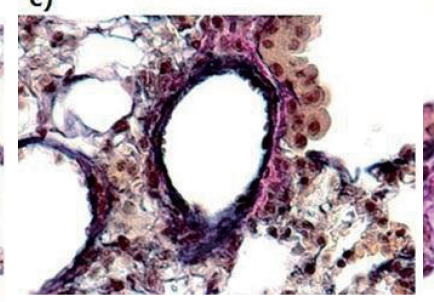

d)

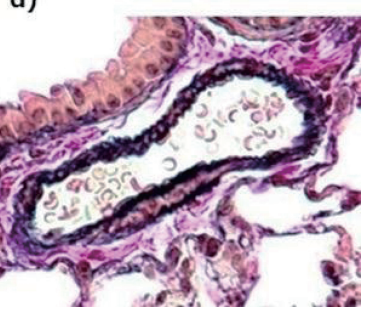

h)

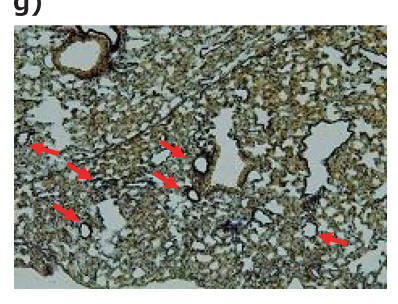

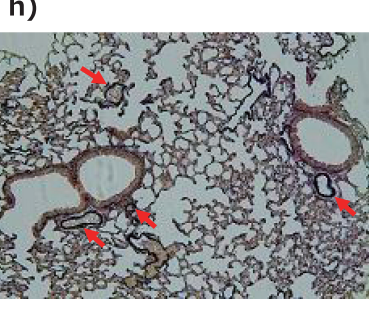

Fig. 2 Histological findings related to pulmonary artery remodeling. a, e) Control group ( $n=5), b, f)$ Monocrotaline-treated PH model group ( $n=5)$. The medial wall of a distal small pulmonary artery was significantly thickened and remarkable fibrosis of the adventitia was observed. c, g) CB-cell group ( $n=5)$. HUCB-MNC transplantation improved medial thickness and fibrosis to levels of those in the control group. $d, h)$ CB-plasma group $(n=5)$. Medial wall thickness and adventitial fibrosis were still as remarkable as those in the PH model group. Lung sections were stained by the elastica van Gieson method (a-d ; x400, e-h ; x100). Red arrows show small pulmonary arteries. Black bar signifies $50 \mu \mathrm{m}$ and $200 \mu \mathrm{m}$ respectively. $\mathrm{PH}$, pulmonary hypertension; $\mathrm{CB}$, cord blood ; hUCB-MNC, human umbilical cord blood-derived mononuclear cell ; $\mathrm{n}$, number of mice. 
a)

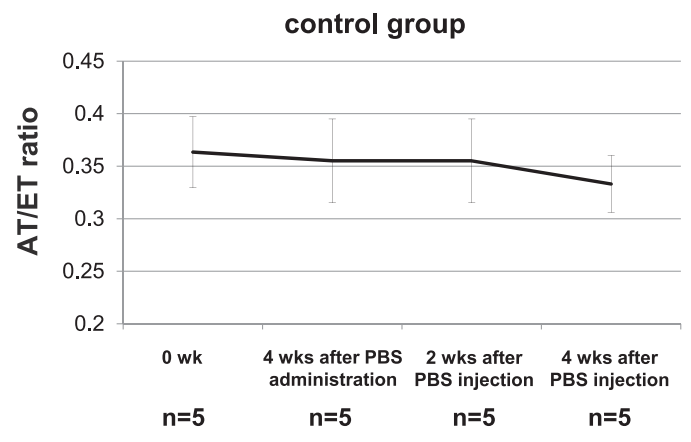

b)

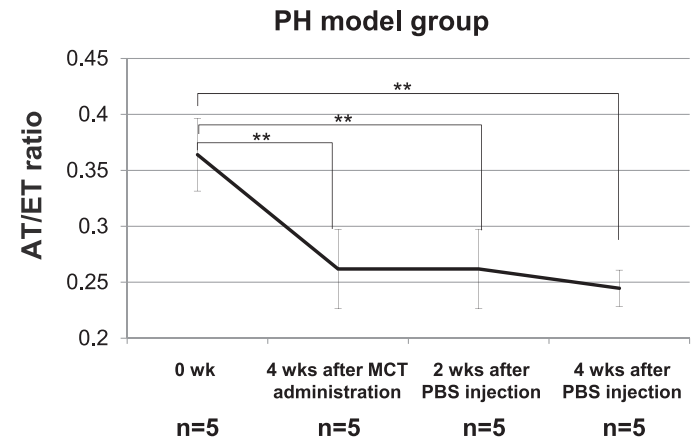

c)

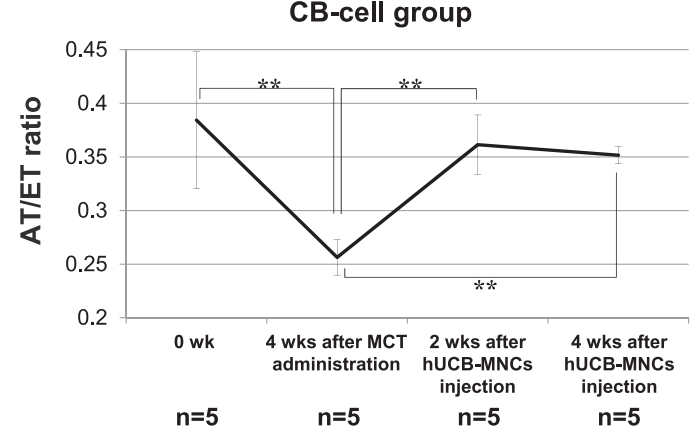

d)

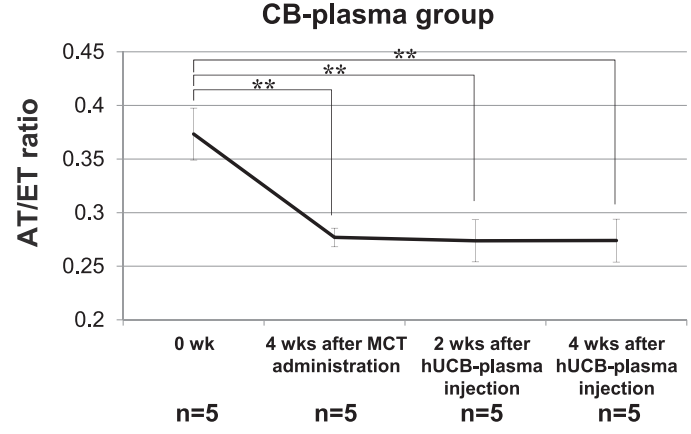

Fig. 4 Changes in the AT/ET ratio calculated from pulmonary blood flow waveform.

a) Vehicle-injected mice (control;n=5) ; b) MCT-treated mice (PH model ; $\mathrm{n}=5$ ) ; c) MCT-treated mice 4 weeks after hUCB-MNC transplantation (CB-cell ; $=5)$; and d) MCT-treated mice 4 weeks after hUCB-plasma injection (CB-plasma ; $\mathrm{n}=5)(* * \mathrm{p}<0.01)$. AT/ET, pulmonary acceleration time to right ventricular ejection time ratio ; $\mathrm{PH}$, pulmonary hypertension ; MCT, monocrotaline ; hUCB-MNCs, human umbilical cord blood-derived mononuclear cells ; CB, cord blood ; n, number of mice. received hUCB-plasma injection without hUCB-cell injection was similar to that in $\mathrm{PH}$ mice. The AT/ET ratio was significantly lower in $\mathrm{PH}$ and $\mathrm{CB}$-plasma mice than in the control mice. On the other hand, the AT/ET ratio was significantly higher in $\mathrm{CB}$-cell mice than in $\mathrm{PH}$ mice.

Detection of Donor Human Umbilical Cord Blood-derived Mononuclear Cells in the Recipient Lung and Other Organs

CMTMR-positive hUCB-MNCs were observed near small pulmonary arterioles and capillaries of the lung from 10 minutes to 3 hours after transplantation (Fig. 5). The number of fluorescently labeled cells in the lung reached a peak at one hour after transplantation and then gradually decreased with the lapse of time.

Intrapulmonary trapping of DiR-labeled hUCB-MNCs was confirmed by IVIS $R$ imaging between 10 minutes and 24 hours after injection (Fig. 6). Until one hour after injection, bioluminescence signals were clearly visible in both lungs and slightly visible in the liver and spleen. After that, the signals in the lungs decreased gradually with passage of time and most of the transplanted hUCB-MNCs were trapped in the liver and spleen. No signal was detected in the heart and intestine. However, the values of fluorescence in the lungs of MCT-treated mice with hUCB-MNC injection were significantly higher at the time of each observation than those of MCTtreated mice with vehicle injection. The values of fluorescence decreased from 10 minutes to 3 hours after hUCB-MNC injection and then remained stable at high levels in the lungs.

\section{DISCUSSION}

In the present study, we observed remarkable improvements in the medial thickness of small pulmonary arteries, RV/S+LV weight ratio and AT/T of pulmonary blood flow after intravenous hUCB-MNC transplantation in a nude mouse model with MCTinduced $\mathrm{PAH}$.

These results demonstrated firstly that hUCB-MNC transplantation alleviated $\mathrm{PH}$ in MCT-induced mice that depended on the presence of intravenously transplanted hUCB-MNCs and secondly that some transplanted hUCB-MNCs were incorporated into the lung tissue around small pulmonary arterioles three hours after transplantation with consequent residence of those cells being significantly observed within 24 hours after transplantation. Bimodal imaging by CMTMR and IVISR spectrum revealed limited graft survival and intrapulmonary cell trapping.

With regard to detection of donor BM-derived MNCs in the recipient lung, both affirmative and negative findings have been reported. Zhao and colleagues showed in a study using a rat model in 2005 that transplanted BM-derived cells survived for at least seven days after delivery and were incorporated and engrafted in the lung tissue and thereby contributed to repair of the pulmonary microvascular structure (18). Although we were previously unable to detect transplanted BM-MNCs labeled with the fluorescent dye carbocyanine in lung tissue at 3 hours, 24 hours, and 1 week after $\mathrm{BM}-\mathrm{MNC}$ transplantation despite improvement of $\mathrm{PH}$ in a MCTinduced mouse model, the discrepancy regarding detection in the two studies may be due to the difference in precision of previous and current imaging modalities (5).

Umbilical cord blood is a readily available source of heterogeneous stem cells that contains both hematopoietic and nonhematopoietic tissue precursors : mesenchymal stem cells and endothelial progenitor cells. Ex vivo work showed the ability of such cells to proliferate in culture and differentiate into a variety of cell types, and homing experiments have demonstrated long-term engraftment of hematopoietic stem cells in xenogeneic ovine and murine models. Transplantation of human cord blood stem cells into an immunosuppressed large animal model, in which long-term 


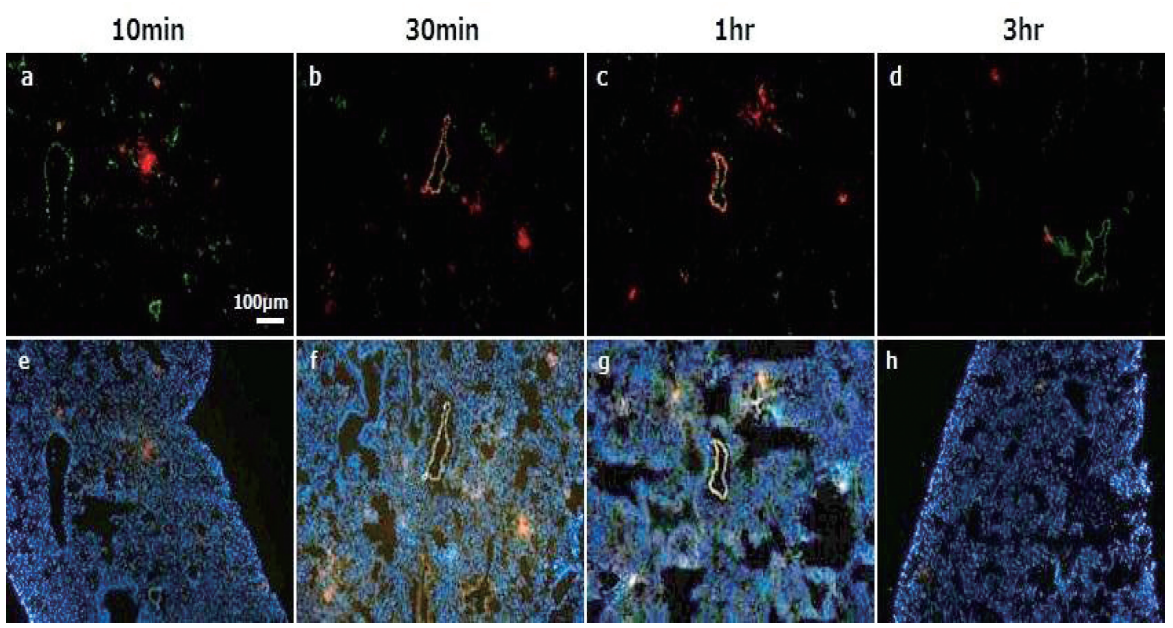

Fig. 5 Assessment of the incorporation of transplanted umbilical cord blood-derived mononuclear cells into the lung.

Panels a- $d$ show low magnification fluorescent micrographs of lungs explanted at $10 \min (n=5), 30 \min (n=5), 1 \mathrm{hr}(n=5)$ and $3 \mathrm{hr}(\mathrm{n}=5)$ after delivery of CMTMR-labeled cells, respectively $(\mathrm{x} 100)$. Red fluorescence represents CMTMR-labeled hUCB-MNCs and green fluorescence indicates vWFpositive vascular endothelium. White bar signifies $100 \mu \mathrm{m}$. Panels e-h show low magnification fluorescent micrographs by DAPI staining of lungs. CMTMR, chloromethyl tetramethylrhodamine ; hUCB-MNCs, human umbilical cord blood-derived mononuclear cells ; vWF, von Willebrand factor ; n, number of mice.

multi-lineage human chimerism has been shown, allows the detection of engraftment (19).

The effectiveness of some cellular therapies, particularly intravenous transplantation of MNCs including endothelial progenitor cells, for PH has been investigated in detail, and a basic understanding of the mechanisms has changed from so-called vasculogenesis originating from endothelial progenitor cells to a paracrine effect by transplanted MNCs $(1-5,11,20)$. Initially, a general mechanism of those therapeutic effects were thought to be via serial steps of incorporation of transplanted or circulating endothelial progenitor cells into the injured lung tissue, differentiation and proliferation for new blood vessel formation (21-23). However, different possible mechanisms relevant to cell transplantation therapies have recently been proposed. O'Neill and associates suggested that BM-derived cells enhance the angiogenic response through paracrine release of growth factors to hypoxia without transdifferentiation into endothelial cells (24-26). It was recently reported that CD34+ exosomes might represent a significant component of the paracrine effect of progenitor cell transplantation for therapeutic angiogenesis (27).

Taking into account those new knowledge, transplanted hUCBMNCs do not pass through the lungs but stay in the lung tissue for at least 24 hours after transplantation and that an appropriate dose of hUCB-MNCs may provide improvement of $\mathrm{PH}$ through active involvement of these cells and modulating the host paracrine effect as an inflammatory response.

\section{Study Limitations}

Firstly, a xenogeneic transplantation rodent model was used in this study. If possible, additional experiments using large animals such as pigs or dogs should be carried out to obtain reliable information relevant to the clinical situation for $\mathrm{PAH}$.

Secondly, we measured neither the actual right ventricle pressure nor pulmonary artery pressure directly in each mouse because of technical problems. We only confirmed indirectly the improvement of $\mathrm{PH}$ through the ratio of weight of myocardium, echocardiographic findings, and histological findings. Actual measurements of pulmonary artery pressure should be used for demonstrating MCT-induced PH and its improvement by hUCB-MNC transplantation.
Finally, the precise working mechanism of hUCB-MNC transplantation for pulmonary artery remodeling remains to be elucidated, for example, whether hUCB-MNCs directly or indirectly modulate vascular function and metabolism. The fate and activity of the inoculated hUCB-MNCs in mice should be revealed with time. Since our data showed that the number and the interval of incorporation of transplanted hUCB-MNCs into the MCT-induced PH mouse lung seemed to be limited, the so-called paracrine/ autocrine mechanism by local secretion of cytokines/chemokines and activation of intracellular cell signaling pathways consequent to hUCB-MNC transplantation may underlie both the longevity and intensity of the improvement of $\mathrm{PH}$. To reveal the possible mechanism between the small number of fluorescently labeled hUCB-MNCs in the lung which reached a peak at as early as at one hour after transplantation and the mechanism by which single hUCB-MNC transplantation dramatically ameliorated pulmonary hypertension at 4 weeks, more sophisticated research by using specific antibodies for human vascular cells and other cellular components will be needed.

Also to understand the basal mechanisms involved in the hUCBMNC-mediated phenotypic changes and the level of interstitial fibrosis, further analyses for the interaction between hUCB-MNC and vascular function in the process of EC dysfunction and the development of vascular diseases are indispensable.

\section{CONCLUSIONS}

HUCB-MNC transplantation may provide a new therapeutic potential for patients with severe $\mathrm{PAH}$ and/or primitive hypoplastic pulmonary vasculature. Furthermore, if restoration therapy using unpurified populations of hUCB-MNCs can be used in patients with $\mathrm{PAH}$, it would be very useful for treating the disease having limited long-term therapeutic options.

\section{ACKNOWLEDGEMENTS}

This study was supported by Grants-in-Aid for Scientific Research from the Japan Society for Promotion of Science (\#24390331). 
a)

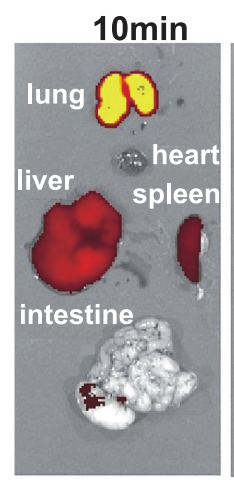

$3 \mathrm{hr}$

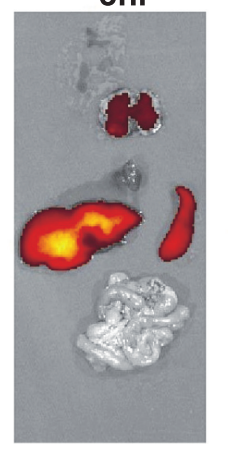

b)

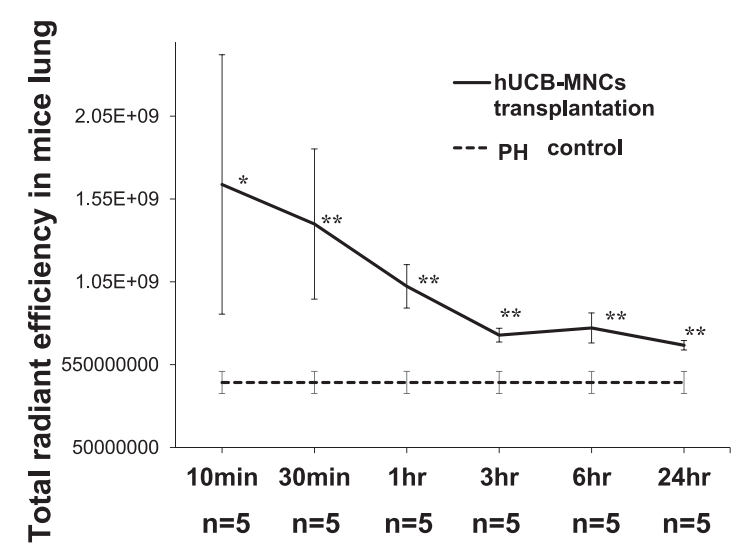

Fig. 6 Assessment of intrapulmonary trapping of DiR-labeled hUCBMNCs by bioluminescence imaging.

a) Bioluminescence images of extracted organs including the lung, heart, liver, spleen, and intestine at $10 \mathrm{~min}(\mathrm{n}=5), 30 \mathrm{~min}(\mathrm{n}=5), 1 \mathrm{hr}$ $(\mathrm{n}=5), 3 \mathrm{hr}(\mathrm{n}=5), 6 \mathrm{hr}(\mathrm{n}=5)$ and $24 \mathrm{hr}(\mathrm{n}=5)$ after injection of hUCBMNCs. N, number of mice.

b) ROI values of the lung after hUCB-MNC transplantation for monocrotaline-induced $\mathrm{PH}$ mice and those after vehicle injection for $\mathrm{PH}$ mice are shown as photons per second/microwatts per square centimeter. $\mathrm{PH}$, pulmonary hypertension ; HUCB-MNCs, human umbilical cord bloodderived mononuclear cells ; $\mathrm{n}$, number of mice. $(* \mathrm{P}<0.05 ; * * \mathrm{p}<0.01)$

\section{DISCLOSURES}

The authors have declared that no conflict of interest exists.

\section{REFERENCES}

1. Takahashi M, Nakamura T, Toba T, Kajiwara N, Kato H, Shimizu Y : Transplantation of endothelial progenitor cells into the lung to alleviate pulmonary hypertension in dogs.
Tissue Eng $10: 771-779,2004$

2. Wang XX, Zhang FR, Shang YP, Zhu JH, Xie XD, Tao QM, $\mathrm{Zhu} \mathrm{JH}$, Chen JZ: Transplantation of autologous endothelial progenitor cells may be beneficial in patients with idiopathic pulmonary arterial hypertension : a pilot randomized controlled trial. J Am Coll Cardiol 49 : 1566-1571, 2007

3. Spees JL, Whitney MJ, Sullivan DE, Lasky JA, Laboy M, Ylostalo J, Prockop DJ : Bone marrow progenitor cells contribute to repair and remodeling of the lung and heart in a rat model of progressive pulmonary hypertension. FASEB J 22 : 1226-1236, 2008

4. Hayashida K, Fujita J, Miyake Y, Kawada H, Ando K, Ogawa S, Fukuda K: Bone marrow-derived cells contribute to pulmonary vascular remodeling in hypoxia-induced pulmonary hypertension. chest $127: 1793-1798,2005$

5. Yoshida H, Kitaichi T, Urata M, Kurobe H, Kanbara T, Motoki T, Kitagawa T: Syngeneic bone marrow mononuclear cells improve pulmonary arterial hypertension through vascular endothelial growth factor upregulation. Ann Thorac Surg 88 : 418-424, 2009

6. Garbuzova-Davis S, Sanberg CD, Kuzmin-Nichols N, Willing AE, Gemma C, Bickford PC, Miller C, Rossi R, Sanberg PR : Human umbilical cord blood treatment in a mouse model of ALS : optimization of cell dose. PLoS One 25 ; 3(6) : e2494, 2008

7. Reems JA, Hall KM, Gebru LH, Taber G, Rich IN : Development of a novel assay to evaluate the functional potential of umbilical cord blood progenitors. Transfusion $48: 620-628$, 2008

8. Wu KH, Zhou B, Mo XM, Cui B, Yu CT, Lu SH, Han ZC, Liu YL: Therapeutic potential of human umbilical cord-derived stem cells in ischemic diseases. Transplant Proc 39 : 16201622,2007

9. Murohara T, Ikeda H, Duan J, Shintani S, Sasaki Ki, Eguchi H, Onitsuka I, Matsui K, Imaizumi T : Transplanted cord bloodderived endothelial precursor cells augment postnatal neovascularization. J Clin Invest 105 : 1527-1536, 2000

10. Nakagawa R, Watanabe T, Kawano Y, Kanai S, Suzuya H, Kaneko M, Watanabe H, Okamoto Y, Kuroda Y, Nakayama T; Chugoku-Shikoku Cord Blood Bank : Analysis of maternal and neonatal factors that influence the nucleated and CD34+ cell yield for cord blood banking. Transfusion 44 : 262-267, 2004

11. Raoul W, Wagner-Ballon O, Saber G, Hulin A, Marcos E, Giraudier S, Vainchenker W, Adnot S, Eddahibi S, Maitre B : Effects of bone marrow-derived cells on monocrotaline- and hypoxia-induced pulmonary hypertension in mice. Respir Res $8: 8,2007$

12. Davies B, Elwood NJ, Li S, Cullinane F, Edwards GA, Newgreen DF, Brizard CP : Human cord blood stem cells enhance neonatal right ventricular function in an ovine model of right ventricular training. Ann Thorac Surg 89 : 585-593, 2010

13. Pascaud MA, Griscelli F, Raoul W, Marcos E, Opolon P, Raffestin B, Perricaudet M, Adnot S, Eddahibi S : Lung overexpression of angiostain aggravates pulmonary hypertension in chronically hypoxic mice. Am J Respir Cell Mol Biol 29 : 449-457, 2003

14. Thibault HB, Kurtz B, Raher MJ, Shaik RS, Waxman A, Derumeaux G, Halpern EF, Bloch KD, Scherrer-Crosbie M : Noninvasive assessment of murine pulmonary arterial pressure : validation and application to models of pulmonary hypertension. Circ Cardiovasc Imaging 3 : 157-163, 2010

15. Zhao YD, Courtman DW, Ng DS, Robb MJ, Deng YP, Trogadis J, Han RN, Stewart DJ : Microvascular regeneration in established pulmonary hypertension by angiogenic gene transfer. 
Am J Respir Cell Mol Biol 35 : 182-189, 2006

16. Everaert BR, Bergwerf I, De Vocht N, Ponsaerts P, Van Der Linden A, Timmermans JP, Vrints CJ : Multimodal in vivo imaging reveals limited allograft survival, intrapulmonary cell trapping and minimal evidence for ischemia-directed BMSC homing. BMC Biotechnol 12 : 93, 2012

17. Oh HJ, Hwang do W, Youn $\mathrm{H}$, Lee DS : In vivo bioluminescence reporter gene imaging for the activation of neuronal differentiation induced by the neuronal activator neurogenic 1 (Ngn1) in neuronal precursor cells. Eur J Nucl Med Mol Imaging 40 : 1607-1617, 2013

18. Zhao YD, Courtman DW, Deng Y, Kugathasan L, Zhang Q, Stewart DJ : Rescue of monocrotaline-induced pulmonary arterial hypertension using bone marrow-derived endotheliallike progenitor cells : efficacy of combined cell and eNOS gene therapy in established disease. Circulation Research 96 : 442-450, 2005

19. Shimizu Y, Ogawa M, Kobayashi M, Almeida-Porada G, Zanjani ED : Engraftment of cultured human hematopoietic cells in sheep. Blood 91(10) : 3688-3692, 1998

20. Losordo DW, Dimmeler S : Therapeutic angiogenesis and vasculogenesis for ischemic disease. Part II : cell-based therapies. Circulation 109 : 2692-2697, 2004

21. Asahara T, Murohara T, Sullivan A, Silver M, van der Zee R, Li T, Witzenbichler B, Schatteman G, Isner JM : Isolation of putative progenitor endothelial cells for angiogenesis. Science 275 : 964-966, 1997

22. Kocher AA, Schuster MD, Szabolcs MJ, Takuma S, Burkhoff D, Wang J, Hommas S, Edwards NM, Itescu S : Neovascularization of ischemic myocardium by human bone-marrow-derived angioblast prevents cardiomyocyte apoptosis, reduces remodeling and improves cardiac function. Nat Med $7: 430-436$, 2001

23. Asahara T, Masuda H, Takahashi T, Kalka C, Pastore C, Silver M, Kearne M, Magner M, Isner JM : Bone marrow origin of endothelial progenitor cells responsible for postnatal vasculogenesis in physiological and pathological neovascularization. Circ Res 85 : 221-228, 1999

24. O'Neil TJ, Wamhoff BR, Owens GK, Skalak TC : Mobilization of bone marrow-derived cells enhances the angiogenic response to hypoxia without transdifferentiation into endothelial cells. Circulation Research 97 : 1027-1035, 2005

25. Campbell AI, Zhao Y, Sandhu R, Stewart DJ : Cell-Based Gene Transfer of Vascular Endothelial Growth Factor Attenuates Monocrotaline-Induced Pulmonary Hypertension. Circulation $104:$ 2242-2248, 2001

26. Iwasaki H, Kuwamoto A, Ishikawa M, Oyamada A, Nakamori S, Nishimura H, Sadamoto K, Horii M, Matsumoto T, Murasawa S, Shibata T, Suehiro S, Asahara T : Dose-Dependent Contribution of CD34-Positive Cell Transplantation to Concurrent Vasculogenesis and Cardiomyogenesis for Functional Regenerative Recovery After Myocardial Infarction. Circulation $113: 1311-1325,2006$

27. Sahoo S, Klychko E, Thorne T, Misener S, Schultz KM, Millay M, Ito A, Liu T, Kamide C, Agrawal H, Perlman H, Qin G, Kishore R, Losordo DW : Exosomes from human CD34+ stem cells mediate their proangiogenic paracrine activity. Circulation Research 109 : 724-728, 2011 